\title{
Structure of titanium alloy, modified by electron beams and destroyed during fatigue
}

\author{
S. V. Konovalov ${ }^{1, \dagger}$, I. A. Komissarova², D. A. Kosinov², Yu. F. Ivanov \\ O. V. Ivanova ${ }^{4}$, V. E. Gromov ${ }^{2}$ \\ tksv@ssau.ru \\ ${ }^{1}$ Samara National Research University, 34 Moskovskoye Shosse, 443086, Samara, Russia \\ ${ }^{2}$ Siberian State Industrial University, 42 Kirov st., 654007, Novokuznetsk, Russia \\ ${ }^{3}$ Institute of High Current Electronics SB RAS, 2/3 pr. Academic, 6342021, Tomsk, Russia \\ ${ }^{4}$ Tomsk State University of Architecture and Building, 2 Solyanaya sq., 634003, Tomsk, Russia
}

\begin{abstract}
The present work is aimed to study the fracture surface and state of the defect substructure after high-cycle fatigue failure of titanium alloy VT1-0 preliminarily subjected to an intense pulsed electron beam irradiation with an electron energy $16 \mathrm{keV}$, pulse rate $0.3 \mathrm{~s}^{-1}$, pulse duration $150 \mu \mathrm{s}$, beam energy flux density $30 \mathrm{~J} / \mathrm{cm}^{2}$ and the number of pulses 3 . Electron beam irradiation led to an enhancement of the fatigue life by $20 \%$ on average with respect to that of unirradiated samples. It has been established that the fatigue fracture has a multilayered character and is characterized by the presence of a surface layer of 20 to $25 \mu \mathrm{m}$ thickness, an intermediate layer of $50-55 \mu \mathrm{m}$ thickness and the bulk of the material. In the surface layer, a sublayer characterized by the presence of micropores adjacent to the irradiation surface can be distinguished. In order to compare with experimental results, the temperature field has been theoretically calculated. The analysis has shown that irradiation of titanium is accompanied by the formation of a relatively thin (about $25 \mu \mathrm{m}$ ) surface layer, which is formed as a result of rapid crystallization. It is shown that the defect substructure of the surface layer in samples failed by fatigue tests consists of a polycrystalline structure based on $\alpha$-Ti; in the volume of grains, a dislocation substructure is observed, represented by randomly distributed dislocations or dislocations forming networks. It is established that the structure of samples irradiated by electron beam and failed by fatigue tests significantly differs from the structure revealed in unirradiated titanium samples by a subgrain structure in the volumes of grains in a surface layer with the thickness of $5 \mu \mathrm{m}$. On a larger distance from irradiated surface (about 20 to $25 \mu \mathrm{m}$ ), a plate-like substructure is observed in the grains. The state of plate-like structure essentially depends on a distance from the irradiated surface. Namely, a transition from a mixed subgrain and plate-like structure to the plate-like one occurs.
\end{abstract}

Keywords: structure, titanium alloy VT1-0, electron-beam treatment, high cycle fatigue.

\section{Структура титанового сплава, модифицированного электронными пучками и разрушенного при усталости}

\author{
Коновалов С. В. ${ }^{1, \dagger}$, Комиссарова И. А. ${ }^{2}$, Косинов Д. А. ${ }^{2}$, Иванов Ю. Ф. ${ }^{3}$, \\ Иванова О. В. ${ }^{4}$, Громов В.Е. ${ }^{2}$ \\ ${ }^{1}$ Самарский национальный исследовательский университет им. академика С.П. Королева, ул. Московское шоссе 34 , \\ 443086, Самара, Россия \\ ${ }^{2}$ Сибирский государственный индустриальный университет, ул. Кирова 42, 654007, Новокузнецк, Россия \\ ${ }^{3}$ Институт сильноточной электроники СО РАН, пр. Академический 2/3, 6342021, Томск, Россия \\ ${ }^{4}$ Томский государственный архитектурно-строительный университет, пл. Соляная 2, 634003, Томск, Россия
}

Выполнено исследование поверхности разрушения и состояния дефектной субструктуры разрушенного при многоцикловой усталости титанового сплава ВТ1-0, подвергнутого предварительно облучению интенсивным импульсным электронным пучком (энергия электронов 16 кэВ; частота следования импульсов $0,3 \mathrm{c}^{-1}$; длительность импульса пучка электронов 150 мкс; плотность энергии пучка электронов 30 Дж/см²; количество импульсов воздействия 3). Облучение электронным пучком привело к увеличению усталостной долговечности в среднем на $20 \%$ относительно необлученных образцов. Установлено, что усталостный излом имеет многослойное строение и характеризуется 
наличием поверхностного слоя толщиной $(20$ - 25) мкм, промежуточного слоя толщиной (50 - 55) мкм и основного объема материала. В поверхностном слое можно выделить подслой, примыкающий к поверхности облучения, характеризующийся наличием микропор. Для сопоставления экспериментальных результатов с данными теоретических расчетов выполнено математическое решение задачи о нахождении температурного поля и установлено, что облучение титана сопровождается образованием тонкого (25,2 мкм) поверхностного слоя, образующегося в результате высокоскоростной кристаллизации расплава. Показано, что дефектная субструктура поверхностного слоя в образцах, разрушенных в результате усталостных испытаний, состоит из поликристаллической структуры на основе $\alpha$-Ті; в объеме зерен наблюдается дислокационная субструктура, представленная хаотически распределенными дислокациями и дислокациями, формирующими сетки. Установлено, что структура образцов, облученных электронным пучком и разрушенных в результате усталостных испытаний, существенно отличается от структуры, выявленной в материале, не подвергнутом облучению: в объеме зерен поверхностного слоя толщиной 5 мкм обнаружена субзеренная структура. На большем удалении от поверхности облучения в слое толщиной $(20-25)$ мкм в объеме зерен выявляется субструктура пластинчатого типа. Состояние пластинчатой структуры существенным образом зависит от расстояния от поверхности облучения электронным пучком: происходит переход от смешанной субзеренно-пластинчатой структуры к структуре пластинчатого типа.

Ключевые слова: структура, титановый сплав ВТ1-0, электронно-пучковая обработка, многоцикловая усталость.

\section{1. Введение}

Титановые сплавы являются одними из широко применяемых в промышленности материалов, которые благодаря уникальному сочетанию таких физических и механических характеристик, как высокая удельная прочность, достаточная технологическая пластичность и высокая коррозионная стойкость, являются одними из наиболее привлекательных конструкционных материалов [1]. Однако расширению области их применения препятствуют низкое сопротивление абразивному и эрозионному изнашиванию, низкая твердость, склонность к схватыванию при работе в узлах трения и низкая износостойкость [2]. Также титановые сплавы являются чувствительными к состоянию поверхности. Это связывается с тем, что после механической обработки при изготовлении образцов в поверхностном слое формируются риски, которые оказывают влияние на их долговечность [3]. Поэтому состояние поверхностного слоя оказывает существенное влияние на усталостную долговечность материала т. к. в большинстве случаев причиной усталостного разрушения металлов и сплавов является наличие концентраторов напряжений и зарождение трещин в поверхностном слое детали [4-6]. Изменение состояния материала на поверхности детали способно существенно улучшить ее свойства и оказать значительное влияние на сопротивление усталости массивных образцов [7].

Апробированных методов нанесения изменения состояния поверхности металлических (в том числе и титановых) изделий в настоящее время существует достаточное количество (микродуговое оксидирование, аэротермоакустическая обработка, ионное азотирование и перемешивание, упрочнение фазовыми составляющими твердых эвтектик, сварочные методы, электровзрывное легирование и др.). Применение методов поверхностного упрочнения приводит к существенному повышению механических характеристик материала, что связано с удалением концентраторов напряжений, сформировавшихся вследствие механической обработки, созданием в поверхностном слое обработанной детали сжимающих остаточных напряжений, дисперги- рованием структуры матрицы и включений вторых фаз [7-14].

Эффективным методом улучшения поверхностного слоя материала является обработка поверхности детали интенсивным импульсным электронным пучком субмиллисекундной длительности воздействия, позволяющим модифицировать структуру поверхностного слоя толщиной в десятки микрометров, переводя его в многослойное структурно-фазовое состояние и, при этом, практически не изменяя структуру и свойства основного объема сплава [15].

Целью настоящей работы являлось установление влияния облучения интенсивным импульсным электронным пучком титанового сплава ВТ1-0 на его усталостную долговечность, а также исследования по сопоставлению данных, полученных методами теоретического моделирования и просвечивающей и сканирующей электронной микроскопии.

\section{2. Материалы и методики}

В качестве материала исследований были использованы образцы титанового сплава ВТ1-0 (аналог - сплав Grade2) химического состава: до 0,18 Fe; до 0,07 C; до 0,04 N; до 0,1 Si; до 0,12 О; до 0.004 Н; 0.3 - другие примеси; остальное $\mathrm{Ti}$, масс. \%). Образцы толщиной 4 мм и шириной 12 мм были изготовлены по ГОСТ 25.502 - 79 и имели симметричный концентратор напряжений с минимальным сужением в центральной части 5 мм. Внешний вид образцов не отличался от приведенных в [16]. Образцы были изготовлены из цельного листа толщиной 4 мм, поставляемого от производителя, путем токарной обработки.

Облучение поверхности образцов, приготовленных для усталостных испытаний, осуществляли на установке «СОЛО» [17 - 19] при следующих параметрах: энергия электронов 16 кэВ; частота следования импульсов $0,3 \mathrm{c}^{-1}$; длительность импульса пучка электронов 150 мкс; плотность энергии пучка электронов 30 Дж/см²; количество импульсов воздействия 3.

Усталостные испытания проводили на установке для испытаний на усталость по схеме асимметричного 
консольного изгиба. Температура испытаний $-300 \mathrm{~K}$, частота нагружения образцов изгибом составляла 10 Гц. Испытания выполнены не менее чем с 10 образцами в каждом состоянии. Результаты затем усреднялись и статистически обрабатывались.

Исследования поверхности разрушения осуществляли методами сканирующей электронной микроскопии (Tesla BS-301). Анализ фазового состава и состояния дефектной субструктуры материала выполняли методами просвечивающей электронной дифракционной микроскопии (JEM-2100). «Тонкие фольги» для исследований готовили методом ионного утонения пластинок, вырезанных электроискровым методом из массивного образца параллельно поверхности разрушения на расстоянии 1-3 мкм от ее поверхности. Исследования выполнены с разрушенными образцами, находящимися в двух состояниях: исходное состояние (без обработки) и после обработки импульсными электронными пучками.

Для объяснения влияния импульсного воздействия электронным пучком проведены теоретические исследования по решению задачи о нахождении температурного поля при заданном значении плотности энергии пучка электронов, основанной на решении уравнения теплопроводности. Рассмотрен одномерный случай нагрева и охлаждения пластины толщиной $d$. Система координат выбрана так, что ось $x$ направлена вглубь образца. При $x=0$ задан тепловой поток, а на тыльной стороне пластины теплообмен отсутствует. Основные формулы и алгоритмы, используемые для расчета, приведены в [20-22].

Для решения уравнения теплопроводности использовали метод конечных разностей [22]. Теплофизические характеристики (удельная теплоемкость, плотность, теплопроводность) титана были взяты из справочника [23]. Толщина поверхностного слоя для тепловых расчетов $\mathrm{d}=0.5 \cdot 10^{-3}$ м, время наблюдения 600 мкс. Расчеты выполнялись для интенсивного электронного пучка с режимами, приведенными ранее.

\section{3. Результаты и обсуждение}

Проведение усталостных испытаний показало, что образцы в исходном состоянии разрушались при достижении $(267 \pm 34) \times 10^{3}$ циклов, а образцы, предварительно подвергнутые воздействию электронным пучком, $(319 \pm 33) \times 10^{3}$ циклов. Таким образом, электроннопучковая обработка образцов привела к увеличению усталостной долговечности в среднем на $20 \%$. Для проведения дальнейших исследований были выбраны образцы в двух состояниях - без воздействия электронным пучком и после электронно-пучковой обработки. Данные образцы показали следующее число циклов до разрушения: $233 \times 10^{3}$ и $323 \times 10^{3}$, следовательно, облучение интенсивным импульсным электронным пучком субмиллисекундной длительности воздействия привело к увеличению усталостной долговечности исследуемого материала на 39\% относительно необлученных образцов.

Характерное изображение усталостного излома образца, обработанного интенсивным импульсным элек- тронным пучком, представлено на рис. 1. Анализируя результаты, представленные на рис. 1, необходимо отметить, что усталостный излом имеет многослойное строение и характеризуется наличием поверхностного слоя (слой 1 на рис. 1с) толщиной $(20-25)$ мкм, промежуточного слоя толщиной $(50-55)$ мкм (слой 2 на рис. 1с) и основного объема материала (слой 3 на рис. 1с). В поверхностном слое идентифицируется подслой, примыкающий к поверхности облучения, характеризующийся наличием микропор (слой 1.1 на рис. 1с).

Численное решение задачи о нахождении температурного поля [20,21], формирующегося в поверхностном слое титана, облученного интенсивным электронным пучком, позволило высказать предположение о причинах образования слоистой структуры, выявленной при исследовании изломов, представленных на рис. 1. Установлено, что облучение титана пучком электронов при указанных параметрах приводит к плавлению поверхностного слоя толщиной 25,2 мкм (рис. 2). Данный слой разбивается на два подслоя: поверхностный, толщиной 16,6 мкм, являющийся однофазным (жидкость) (рис. 2, слой 1) и переходный слой толщиной 8,6 мкм, являющийся двухфазным (жидкость + твердое состояние) (рис. 2, слой 2). Время существования первого слоя составляет 128,5 мкс; интервал времени от начала плавления до завершения кристаллизации титана составляет 244,2 мкс.

Таким образом, анализ результатов тепловых расчетов показал, что облучение титана интенсивным электронным пучком с параметрами 30 Дж/см², 150 мкс в одноимпульсном режиме сопровождается образованием сравнительно тонкого ( $\approx 25$ мкм) поверхностного слоя,

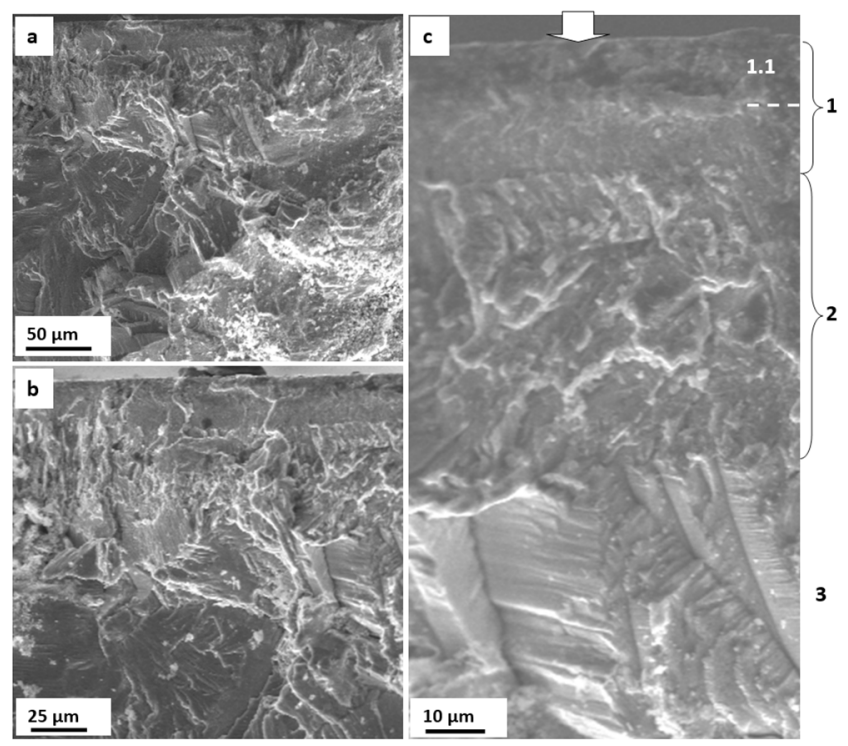

Рис. 1. Структура поверхности разрушения образца, подвергнутого облучению интенсивным электронным пучком и разрушенного при усталостном нагружении. На (c) стрелкой указана поверхность облучения. Сканирующая электронная микроскопия.

Fig. 1. Structure of the fracture surface of a sample subjected to irradiation with an intense electron beam. The (c) arrow indicates the irradiation surface. Scanning electron microscopy. 
образующегося в результате высокоскоростной кристаллизации расплава. Из сопоставления результатов, представленных на рис. 1 и рис. 2, следует, что поверхностный слой 1 (рис. 1) сформировался в результате плавления и скоростной кристаллизации материала; слой 2 (рис. 1) является слоем термического влияния.

На рис. 3 представлены характерные изображения дефектной субструктуры поверхностного слоя материала (в состоянии без обработки электронным пучком), разрушенного в результате усталостных испытаний. Из рис. 3 следует, что исследуемый материал имеет поликристаллическую структуру на основе $\alpha$-титана; в объеме зерен наблюдается дислокационная субструктура, представленная хаотически распределенными дислокациями (рис. 3а) и дислокациями, формирующими нерегулярные сетки (рис. 3b). Скалярная плотность дислокаций, определенная методом секущей [11], составляет $\approx 2,8 \times 10^{10} \mathrm{~cm}^{-2}$. Подобная дислокационная субструктура выявляется в объеме зерен в области, расположенной на расстоянии (150 - 200) мкм от поверхности образца.

Структура поверхностного слоя титана, облученного интенсивным импульсным электронным пучком и разрушенного в результате усталостных испытаний, существенным образом отличается от структуры, выявленной в титане, не подвергнутом предварительному облучению. А именно, по морфологическому признаку можно выделить тонкий поверхностный слой толщиной $\approx 5$ мкм, в объеме зерен которого наблюдается субзеренная структура, характерное изображение которой представлено на рис. 4а. Субзерна имеют глобулярную форму, размеры их изменяются в пределах (500 - 700) нм. На большем удалении от поверхности облучения в слое толщиной (20-25) мкм в объеме зерен выявляется субструктура пластинчатого типа, характерное изображение которой представлено на рис. $4 c, d$. Состояние пластинчатой структуры существенным образом зависит от расстояния от поверхности облучения электронным пучком. А именно, наблюдается переход от смешанной субзеренно-пластинчатой структуры (рис. 4c) к собственно структуре пластинчатого типа (рис. $4 \mathrm{~d}$ ). В объеме субзерен и пластин выявляется сетчатая дислокационная субструктура, характерное изображение которой представлено на рис. $4 \mathrm{~b}$.

Поверхностный слой с пластинчатой субструктурой контактирует с промежуточным слоем, в объеме зерен которого выявляется дислокационная субструктура, представленная хаотически распределенными дислокациями, дислокационными клубками и дислокационными сетками; скалярная плотность дислокаций составляет $1,2 \times 10^{10} \mathrm{~cm}^{-2}$ (рис. 5). Следует отметить, что подобная субструктура выявляется и в зернах разрушенных образцов, не подвергавшихся предварительному облучению интенсивным электронным пучком.

Сопоставляя результаты тепловых расчетов и результаты, полученные методами дифракционной электронной микроскопии, можно заключить, что поверхностный слой с пластинчатой структурой сформировался в результате высокоскоростной кристаллизации титана, имеющей место при электронно-пучковой обработке.

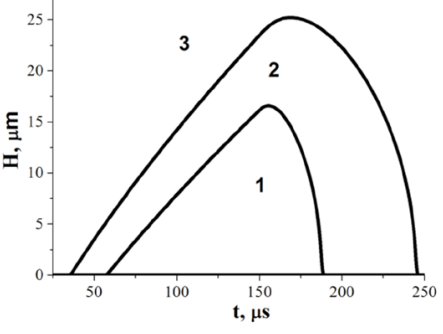

Рис. 2. Зависимость толщины однофазного (жидкость) слоя 1 и двухфазного (твердая фаза + жидкость) слоя 2 от времени наблюдения при облучении поверхности титана электронным пучком с плотностью энергии $\mathrm{E}_{\mathrm{s}}=30$ Дж/ $\mathrm{cm}^{2}$ и длительностью импульса 150 мкс; область 3 - область расположения твердой фазы.

Fig. 2. Thickness of mono-phase (liquid) layer 1 and double-phase (solid phase + liquid) layer 2 vs. time of observation when irradiating the surface of titanium by electron beam with energy density $\mathrm{E}_{\mathrm{s}}=30 \mathrm{~J} / \mathrm{cm}^{2}$ and pulse duration $150 \mu \mathrm{s} ; 3$ is the area of the solid phase. H: thickness of layer $1, \mathrm{t}$ : time of observation.
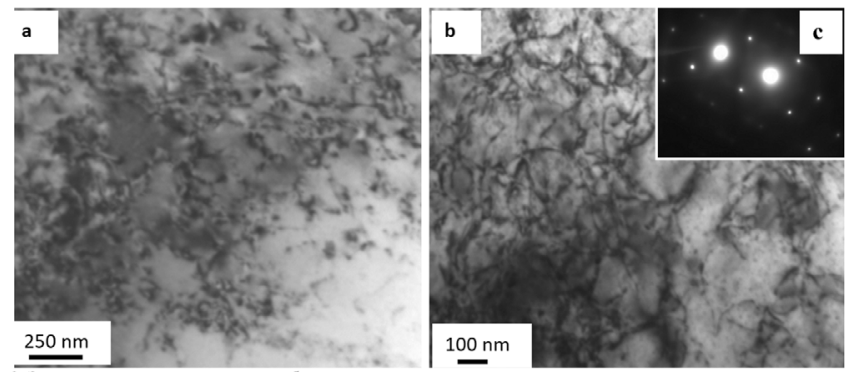

Рис. 3.Электронно-микроскопические изображения структуры поверхностного слоя титанового сплава, разрушенного в результате испытаний на усталостную долговечность; a, b - светлые поля; c - микроэлектронограмма (для b). Просвечивающая дифракционная электронная микроскопия.

Fig. 3. Electron microscopic images of the structure of the surface layer of titanium alloy, destroyed as a result of tests for fatigue life; $\mathrm{a}, \mathrm{b}-$ bright fields; $\mathrm{c}-$ microelectronogram (for b). Transmission diffraction electron microscopy.
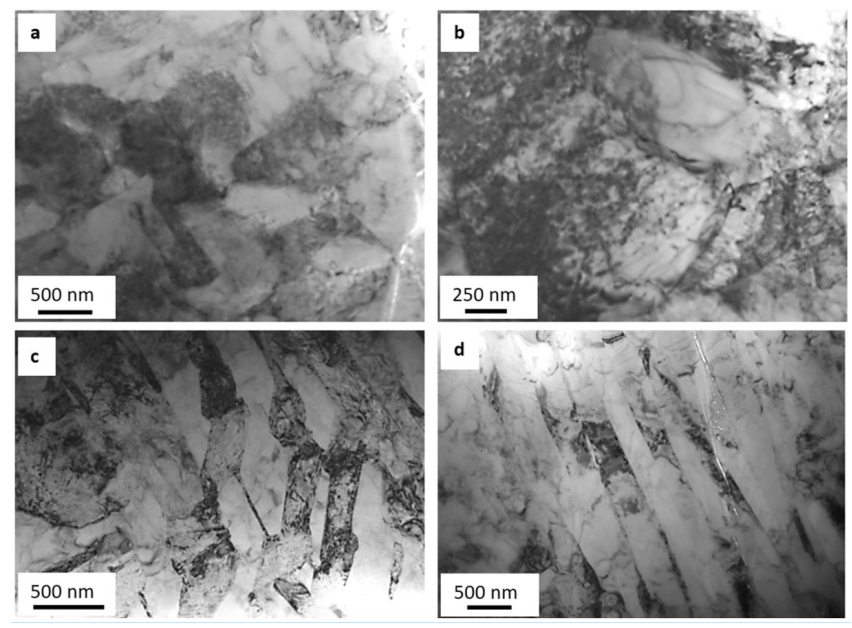

Рис. 4. Электронно-микроскопические изображения структуры титанового сплава, подвергнутого облучению интенсивным электронным пучком и последующим усталостным испытаниям. Поверхностный слой.

Fig. 4. Electron microscopic images of the structure of a titanium alloy subjected to irradiation with an intense electron beam and subsequent fatigue tests. The surface layer. 


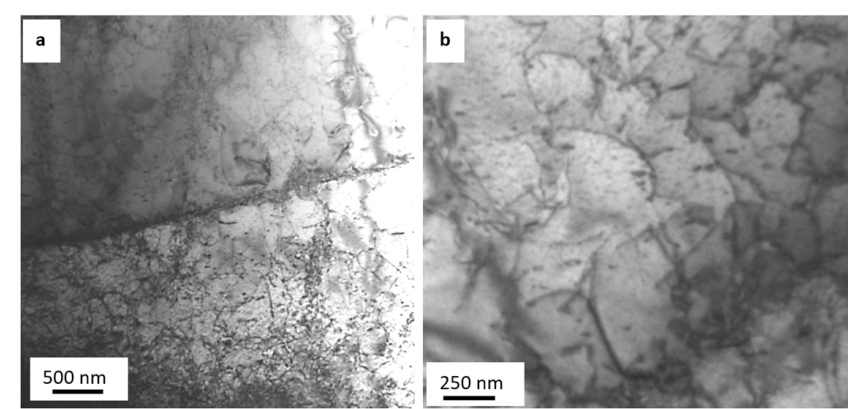

Рис. 5. Электронно-микроскопические изображения структуры титанового сплава, подвергнутого облучению интенсивным электронным пучком и последующим усталостным испытаниям. Промежуточный слой.

Fig. 5. Electron microscopic images of the structure of titanium alloy, subjected to irradiation with an intense electron beam and subsequent fatigue tests. The indermediate layer.

\section{4. Заключение}

1. Показано, что воздействие интенсивным импульсным электронным пучком образцов титанового сплава ВТ1-0 приводит к увеличению их усталостной долговечности в среднем на $20 \%$.

2. Методами сканирующей электронной микроскопии установлено, что разрушенные образцы, предварительно подвергнутые обработке электронными пучками, имеют многослойное строение и характеризуется наличием поверхностного слоя толщиной $(20-25)$ мкм, промежуточного слоя толщиной $(50-55)$ мкм и основного объема материала.

3. Теоретическими расчетами установлено, что облучение титана пучком электронов приводит к образованию тонкого поверхностного слоя толщиной 25,2 мкм, образующегося в результате высокоскоростной кристаллизации расплава.

4. Методами просвечивающей электронной дифракционной микроскопии показано, что структура поверхностного слоя титана, облученного импульсным электронным пучком и разрушенного в результате усталостных испытаний, существенным образом отличается от структуры, выявленной в титане, не подвергнутом предварительному облучению.

Благодарность/Acknowledgements. Исследование выполнено при финансовой поддержке РФФИ (проекmы № 17-32-50003 мол_нр, № 16-32-60048 мол_а_дк и № 16-58-00075 Бел_а) и государственного задания № 3.1283.2017/4.6.

\section{Литература/References}

1. M.H. Mukhametrakhimov. Letters on materials, 5(2), 194 - 197, (2015) (in Russian) [М.X. Мухаметрахимов. Письма о материалах. 5 (2), 194 - 197 (2015)]. DOI: 10. 22226/2410-3535-2015-2-194-197.

2. K.N. Ramazanov, I.S. Ramazanov. Vestnik UGATU. 18(63), 41 - 46 (2014) (in Russian) [К.Н. Рамазанов, И. С. Рамазанов. Вестник УГАТУ. 18 (2), 41 - 46 (2014)].
3. V.I. Betekhtin, A.G. Kadomtsev, M.V. Narykova, M. V. Bannikov, S. G. Abaimov, I. Sh. Akhatov, T. PalinLuc, O.B. Naimark. Phys. Mesomech. 20(1) 78-89 (2017). DOI: 10.1134/S1029959917010076

4. H. Mughrabi, H. Christ. ISIJ International. 37, 1154- 1169 (1997).

5. Y. Ochi, T. Sakai. Zairyo/Journal of the Society of Materials Science. 52, 433 - 439 (2003).

6. L. Lu, W. Zhang. Jixie Qiangdu/Journal of Mechanical Strength. 27, $388-394$ (2005).

7. E.A. Erubaev, Ju.R. Kolobov, I.N. Kuz’menko, G. V. Hramov, M.B. Ivanov, S.S. Manohin. Fundamental'nye issledovanija. 12, 2575-2579 (2014) (in Russian) [Е.А. Ерубаев, Ю.Р. Колобов, И.Н. Кузьменко, Г.В. Храмов, М.Б. Иванов, С. С. Манохин. Фундаментальные исследования. 12, 2575 - 2579 (2014)].

8. V.N. Uskov, G.A. Danilin, G.A. Vorob'eva, A. V. Titov, E. Ju. Remshev, Ju.S. Kukunja. Metalloobrabotka. 1(73), 50 - 54 (2013). (in Russian) [В.Н. Усков, Г. А. Данилин, Г.А. Воробьева, А.В. Титов, Е.Ю. Ремшев, Ю.С. Кукуня. Металлообработка. 1(73), 50-54 (2013)].

9. V.L. Vorob'ev, P. V. Bykov, S.G. Bystrov, A. A. Kolotov, V. Ja. Bajankin, V.F. Kobziev, T. M. Mahneva. Himicheskaja fizika i mezoskopija. 15(4), 576-581 (2013). (in Russian) [В.Л. Воробьев, П.В. Быков, С.Г. Быстров, А.А. Колотов, В.Я. Баянкин, В.Ф. Кобзиев, Т.М. Махнева. Химическая физика и мезоскопия. 15(4), 576-581 (2013)].

10. S. V. Konovalov, V.E. Kormyshev, Y.F. Ivanov, A. D. Teresov. Letters on materials. 6(4), 350 - 354 (2016). DOI: 10.22226/2410-3535-2016-4-350-354.

11. T.Yu. Kobzareva, V.E. Gromov, Yu.F. Ivanov, E. A. Budovskkh, S. V. Konovalov. IOP Conference Series: Materials Science and Engineering. 150(1), 012042 (2016). DOI: 10.1088/1757-899X/150/1/012042

12. O.V. Bashkov, Y.P. Sharkeev, S.V. Panin, V.A. Kim, T.I. Bashkova, A.A. Popkova, A.Y. Eroshenko, A. I. Tolmachev. AIP Conference Proceedings. 1783(10), 020013 (2016). DOI: 10.1063/1.4966306

13. X. Chen, Y. Fang, P. Li, Z. Yu, X. Wu, D. Li. Materials and Design. 65, 1214-1221 (2015). DOI: 10.1016/j. matdes.2014.10.013.

14. I. Y. Timoshkin, K. V. Nikitin, V.I. Nikitin, V.B. Deev. Russian Journal of Non-Ferrous Metals. 57(5), 419-423 (2016). DOI: 10.3103/S1067821216050163

15. A. P. Laskovnev, Ju. F. Ivanov, E. A. Petrikova, N. N. Koval', V. V. Uglov, N. N. Cherenda, N. V. Bibik, V. M. Astashinskij. Modifikacija struktury i svojstv jevtekticheskogo silumina jelektronno-ionno-plazmennoj obrabotkoj. Minsk: «Belorusskaja nauka», 2013. — 287 s. (in Russian) [А.П. Ласковнев, Ю.Ф. Иванов, Е.А. Петрикова, Н.Н. Коваль, В.В. Углов, Н.Н. Черенда, Н. В. Бибик, B.M. Асташинский. Модификация структуры и свойств эвтектического силумина электронно-ионно-плазменной обработкой. - Минск: «Белорусская наука», 2013. - 287 с.]

16. S. Konovalov, I. Komissarova, D. Kosinov, Yu. Ivanov, V. Gromov, O. Semina. Key 
Engineering Materials. 704, 15-19 (2016). DOI: 10.4028/www.scientific.net/KEM.704.15

17. Yu. F. Ivanov, N. N. Koval. Low-energy electron beams submillisekundnoy duration: reception and some aspects of the application in the field of materials science Chapter 13 in the book "Structure and properties of advanced metallic materials." p. 345-382/Ed. A. I. Potekaev. Tomsk: Publishing house of the NTL. (2007) 580 p. (in Russian) [Иванов Ю. Ф., Коваль Н. Н. Низко энергетические электронные пучки субмиллисекундной длительности: получение и некоторые аспекты применения в области материаловедения - Гл.13 в книге «Структура и свойства перспективных металлических материалов». - С.345-382/Под общ. ред. А. И. Потекаева. Томск. НТЛ. 2007. 580 с.]

18. V. A. Grishunin, V. E. Gromov, Y.F. Ivanov, A. D. Teresov, S. V. Konovalov. Journal of Surface Investigation. 7(5), 990 - 995 (2013), DOI: 10.1134/S1027451013050091

19. N. N. Koval', Yu. F. Ivanov. Russian Physics Journal. 51(5),
505- 516 (2008), DOI: 10.1007/s11182-008-9073-7

20. A.A. Samarskii A.A. Introduction to Numerical Methods. Moscow. Nauka (1997). 271 p. (in Russian) [А.А. Самарский. Введение в численные методы. М.: Наука, 1997. 271 с.]

21. V. Rotshtein, Yu. Ivanov, A. Markov. Surface treatment of materials with low-energy, high-current electron beams. Chapter 6 in Book "Materials surface processing by directed energy techniques". - P.205-240. Ed. by Y. Pauleau: Elsevier (2006) 763 p.

22. A. A. Samarskij. Teorija raznostnyh shem. - M.: Nauka, 1989. - 616 s. (in Russian) [А.А. Самарский. Теория разностных схем. - М.: Наука, 1989. - 616 с.]

23. Fizicheskie velichiny: Spravochnik/A.P. Babichev, N.A. Babushkina, A.M. Bratkovskij i dr. - M.; Jenergoatomizdat, 1991. - 1232 s. (in Russian) [Физические величины: Справочник/А.П. Бабичев, Н.А. Бабушкина, А.М. Братковский и др. - М.; Энергоатомиздат, 1991. - 1232 с.] 